1

2

3

4 Milán Szabón a,\#, ${ }^{\star}$, Daniel Wangpraseurt ${ }^{\mathrm{a}, \#}$, Bojan Tamburic ${ }^{\mathrm{a}}$, Anthony W.D. Larkum ${ }^{\mathrm{a}}$, Ulrich Schreiber $^{\mathrm{b}}$

5 David J. Suggett ${ }^{\mathrm{a}}$, Michael Kühl ${ }^{\mathrm{a}, \mathrm{c}}$, Peter J. Ralph ${ }^{\mathrm{a}}$

6

$7 \quad{ }^{a}$ Plant Functional Biology and Climate Change Cluster, University of Technology, Sydney, Broadway 8 2007, NSW, Australia

9 bJulius-von-Sachs Institut für Biowissenschaften, Lehrstuhl Botanik I, Universität Würzburg, Germany

10 Marine Biological Section, Department of Biology, University of Copenhagen, DK-3000 Helsingør,

11 Denmark.

12

13

14

15

16

17

18

19

20

21

22

23

24

damicornis

\section{Effective light absorption and absolute electron transport rates in the coral Pocillopora}

damicornis

(1)

12

\#These authors contributed equally to the present work

*Corresponding Author: Dr. Milán Szabó, Plant Functional Biology and Climate Change Cluster, Faculty of Science, University of Technology, Sydney, 745 Harris St. and 95 Thomas St. Ultimo 2007, NSW, Australia; PO Box 123, Tel.: +61-2-9514-4162, Fax: +61-2-9514-4079, Email:

milan.szabo@uts.edu.au 
$\mathrm{AL}$, Actinic light; $E_{\mathrm{d}}$, Incident downwelling photon irradiance $(400-700 \mathrm{~nm}) ; E_{0}$ (meas), Photon scalar irradiance within coral tissue (measured with microsensors, 400-700 nm); $E_{0}$ (calc), Photon scalar irradiance within coral tissue (calculated based on $\sigma_{\| I}(\lambda)$ ); ETR, Electron transport rate of PS II ( $\mu$ mol electrons $\mathrm{m}^{-2} \mathrm{~s}^{-1}$ ); ETR $\mathrm{E}_{\|}$, Absolute rate of PSII turnover (electrons $\mathrm{PSII}^{-1} \mathrm{~s}^{-1}$ ); $\mathrm{ETR}_{\|}{ }^{\max }$, Maximum rate of PSIl turnover (electrons PSII ${ }^{-1} \mathrm{~s}^{-1}$ ); FRRf, Fast Repetition Rate fluorometry; LC, Light curve of fluorescence parameters, defined as photosynthesis versus irradiance; MC-PAM, Multi-colour Pulse Amplitude Modulated fluorometer, NPQ, Non-photochemical quenching; $\sigma_{\|}(\lambda)$, Wavelength-dependent absorption cross-section of PSII $\left(\mathrm{nm}^{2}\right)$ as determined by the MC-PAM fitting routine

Abstract

Pulse Amplitude Modulation (PAM) fluorometry has been widely used to estimate the relative photosynthetic efficiency of corals. However, both the optical properties of intact corals as well as past technical constrains to PAM fluorometers have prevented calculations of the electron turnover rate of PSII. We used a new multi-colour PAM (MC-PAM) in parallel with light microsensors to determine for the first time the wavelength-specific effective absorption cross-section of PSIl photochemistry, $\sigma_{\|}(\lambda)$, and thus PAM-based absolute electron transport rates of the coral photosymbiont Symbiodinium both in culture and in hospite in the coral Pocillopora damicornis. In both cases, $\sigma_{\|}$of Symbiodinium was highest in the blue spectral region and showed a progressive decrease towards red wavelengths. Absolute values for $\sigma_{\|}$at $440 \mathrm{~nm}$ were up to 1.5-times higher in culture than in hospite. Scalar irradiance within the living coral tissue was reduced by $20 \%$ in the blue when compared to the incident downwelling irradiance. Absolute electron transport rates of $P$. damicornis at $440 \mathrm{~nm}$ revealed a maximum PSII turnover rate of ca. 250 electrons $\mathrm{PSII}^{-1} \mathrm{~s}^{-1}$, consistent with one PSII turnover for every 4 photons absorbed by PSII; this likely reflects the limiting steps in electron transfer between PSII and PSI. Our results show that optical properties of the coral host strongly affect light use efficiency of Symbiodinium. Therefore, relative electron transport rates do not reflect the productivity rates (or indeed how the photosynthesis-light response is parameterised). Here we 
Keywords: chlorophyll fluorescence, electron transport rate, coral, light absorption, spectral attenuation

\section{Introduction}

Coral primary productivity reflects a key indicator of "reef health" (Hoegh-Guldberg et al. 2007) but is time-consuming and labour intensive to measure based on conventional gas-exchange techniques (e.g. Warner et al. 2011) and is further complicated by the close coupling of symbiont photosynthesis and respiration with host respiration (Kühl et al. 1995). Pulse-amplitude modulated (PAM) chlorophyll fluorometry and the Saturation Pulse method (reviewed in Schreiber, 2004) are rapid tools for assessing the photosynthetic capacity of corals both in situ and in the laboratory (Hill et al. 2004; Ralph et al. 1999; Warner et al. 2011). Typically, PAM measurements of coral photosynthesis rely on determination of the effective PSIl photochemical efficiency, $\Delta F^{\prime} / F_{M}$, under known irradiance levels of actinic light $(A L)$, which is often quantified as the incident photon irradiance of photosynthetic active radiation ( $P A R=400-700 \mathrm{~nm}$ ). A relative measure of photosynthetic activity is then calculated in terms of the relative electron transport rates $(r E T R), r E T R=\Delta F / F_{M} \cdot P A R \cdot 0.42$, where $\Delta \mathrm{F} / \mathrm{F}_{\mathrm{M}}$ stands for maximal quantum yield and 0.42 is the so-called "ETR factor", which is a constant value assuming that $84 \%$ of all light is absorbed (as for plant leaves) and that only half of all light absorbed is delivered to PSII for photochemistry (Schreiber et al. 2012). However, the actual amount of light absorbed for PSII photochemistry is unknown for the symbiont algae (Symbiodinium sp.) residing in corals, and also appears variable amongst different genetic types of Symbiodinium sp. and their growth condition (Hennige et al. 2009; Suggett et al. 2011), placing fundamental limitations on applying PAM measurements to yield absolute coral photosynthesis rates.

Light harvesting by PSII is governed by the absorption cross-section of PSIl photochemistry $\left(\sigma_{\mathrm{PSII}}\right)$ and concentration of functional PSII units (Oxborough et al. 2012; Schreiber et al. 2012). Importantly, $\sigma_{\mathrm{PSII}}$ describes an absolute measure of the photon capture efficiency of PSII, which is directly related to the photochemical efficiency of PSII (Ley and Mauzerall, 1982) and thus is indicative of the biochemical nature and biophysical arrangement of pigments within the light harvesting antennae (e.g. Suggett et al. 2004). In the past $\sigma_{\text {PSII }}$ has been determined routinely using fast repetition rate fluorometry (FRRf) or fluorescence induction and relaxation (FIRe) fluorometry (Kolber et al. 1998) that employ single turnover flashes to progressively reduce the primary quinone 
electron acceptor, $Q_{A}$, whereby the rise kinetics from the minimum to maximum fluorescence yield, i.e., from fully oxidised to fully reduced state of $\mathrm{Q}_{\mathrm{A}}$, describes the effective absorption and hence $\sigma_{\mathrm{PSII}}$. To date, measurements of $\sigma_{\mathrm{PSII}}$ were mostly done with 'optically thin' microalgal samples (Koblizek et al. 2001; Schreiber et al. 2012; Schreiber and Klughammer, 2013; Suggett et al. 2004, 2009), including Symbiodinium cultures (Hennige et al. 2009, Ragni et al. 2010). The few published measurements of coral $\sigma_{\mathrm{PSII}}$ values show that $\sigma_{\mathrm{PSII}}$ for Symbiodinium sp. in hospite appears highly dynamic amongst coral species (Gorbunov et al. 2001; Hennige et al. 2011; Levy et al. 2003;), across water depth (Lesser 2000) and daytime (Gorbunov et al. 2001). Based on such PSII cross-sectional area determinations, the rate of photosynthetic electron transport per PSII reaction center was estimated in corals (Gorbunov et al. 2001).

The apparent 'plasticity' of $\sigma_{\mathrm{PSII}}$ in corals could play a key role in moderating absolute electron transfer rates and hence productivity in corals. It likely reflects differences in Symbiodinium genotype dominance since pigment properties (Hennige et al. 2009) and energetic connectivity amongst PSII reaction centres (Ragni et al. 2010, Hennige et al. 2011) is known to vary amongst sub-clades. Whilst these past measurements have demonstrated the inherent variability of $\sigma_{\mathrm{PSII}}$ in corals, they only involved measurements at a single wavelength and were not directly applicable to absolute production estimates without additional knowledge of the spectral dependency of PSII light harvesting. Light absorption by Symbiodinium is highest in the blue region $(440-480 \mathrm{~nm})$ due to the cumulative absorption of the photosynthetic pigments of chlorophyll $a, c_{2}$ and carotenoids. Past FRR and FIRe fluorometers were therefore designed to target peak absorption, and hence $\sigma_{\text {PSIII }}$ in the blue (450-480 nm). Recent developments in chlorophyll fluorescence instrumentation, the Multi-colour Pulse Amplitude Modulated chlorophyll fluorometer (further referred as MC-PAM, Schreiber et al. 2012) however allows the determination of $\sigma_{\mathrm{PSII}}$ at multiple wavelengths across the visible spectral range, i.e also in the green $(\sim 560 \mathrm{~nm})$ that is of special importance in dinoflagellates due to the light absorption by peridinin, located in their light-harvesting pigment-protein antenna complexes (see e.g. Iglesias-Prieto et al. 1993). The measuring principle for determining effective absorption of PSII with the MC-PAM is somewhat different from the approaches used in FRR and FIRe fluorometry (see above) and relies on so-called "O-I $I_{1}$ induction kinetics" (Schreiber et al. 2012). Briefly, O-I kinetics describe the photochemical phase in the polyphasic rise of PSII fluorescence at the onset of strong actinic illumination used to yield a $\mathrm{O}-\mathrm{I}_{1}-\mathrm{I}_{2}-\mathrm{P}$ induction curve (Schreiber et al. 2004); another 
nomenclature describes this as the O-J-I-P induction curve (Strasser and Govindjee, 1992). Both $\mathrm{I}_{1}$ and $\mathrm{J}$ fluorescence yields mark the end of the photochemical and the start of the thermal induction phase but their determination and interpretation are somewhat different (Schreiber et al. 2012). $I_{1}$ is determined via a saturating single turnover flash applied at the end of the photochemical phase, which is driven by continuous actinic light (AL or MT). Following the saturating single turnover flash, $Q_{A}$ is first fully reduced and then partially reoxidized with a time constant in the order of $300-400 \mu \mathrm{s}$. The $I_{1}$-level is determined by extrapolation to the end of the flash. While $J$ is also determined at the end of the photochemical phase, it simply represents a single inflection or step in the induction curve, where light driven $Q_{A}$ reduction and reoxidation via electron transfer to the secondary acceptor $Q_{B}$ overlap; therefore, fluorescence yield corresponding to full reduction of $Q_{A}{ }^{-}$cannot be precisely determined without application of a flash (Schreiber et al. 2012). Accurate analysis of the O-I $I_{1}$ kinetics requires that changes in the original variable fluorescence yield are transformed into changes in $Q_{A}$ reduction (PSIl closure) by the equation $\left(F-F_{0}\right) /\left(I_{1}-F_{0}\right)=(1-q) /(1+J q)$, with $(1-q)$ representing the fraction of closed PSII reaction centres and $J$ being a measure of connectivity between PSII units (Lavergne and Trissl 1995).

The alternative measuring and analysis routines used in the MC-PAM as compared to FRR (FIRe) fluorometry requires specification of alternate terminology for the wavelength-dependent absorption cross-section of PSII, which was defined here as $\sigma_{\| I}(\lambda)$ (Schreiber and Klughammer, 2013). Using FRR/FIRe fluorometry, the $\sigma_{\mathrm{PSII}}(\lambda)$ can be determined for any physiological state, notably different states of PSII reaction centre openness, redox conditions in the intersystem electron transport chain, membrane energisation, and state 1/state 2 conditions of the photosynthetic apparatus (e.g. Oxborough et al. 2012). It should be noted, however, that if $\sigma_{\text {PSII }}(\lambda)$ information is combined with information on the effective PS II quantum yield to estimate electron transport rate, care must be taken that e.g. NPQ by membrane energisation is not effective twofold by lowering PS II cross section as well as PS II quantum yield. In contrast, $\sigma_{\|}(\lambda)$ as defined for measurements with the MC-PAM is only defined for a particular reference state, where $Q_{A}$ and the plastoquinone (PQ) pool are oxidized, and non-photochemical quenching (caused by membrane energisation or state 2 formation) is absent (Schreiber et al. 2012). Thus, for calculation of electron transport rates, any loss 
open reaction centres by non-photochemical quenching mechanisms is not accounted for via $\sigma_{\| I}(\lambda)$ but via the measured effective PSIl photochemical efficiency, $\Delta F / F_{M}$ (Schreiber et al. 2011, 2012; see also Suggett et al. 2011). In our study, $\sigma_{\|}(\lambda)$ refers to the intrinsic, maximal potential absorption crosssection of PSII at a given wavelength, measured under the same conditions under which the PSII photochemical efficiency is at its maximum (i.e., $\Delta \mathrm{F} / \mathrm{F}_{\mathrm{M}}$ ). In its original definition, this intrinsic $\sigma_{\text {II }}(\lambda)$ can be determined in optically thin samples only, i.e., in the absence of wavelength-dependent light gradients. However, the present study explores its possible application in opaque samples such as thin-tissued corals. In surface-associated phototrophs such as corals, the optical properties of the host system strongly modulate the actual light availability of zooxanthellae in hospite (Enriquez et al. 2005; Kühl et al. 1995; Teran et al. 2010; Wangpraseurt et al. 2012, 2014). Coral tissue and the coral skeleton are both strong scatterers (Marcelino et al. 2013; Wangpraseurt et al. 2014), and as scattering increases the path length of photons per vertical distance travelled it can lead to a local enhancement in scalar irradiance over the incident downwelling irradiance (Wangpraseurt et al. 2012). Steep light gradients exist within the coral tissue, where scalar irradiance can attenuate by one order of magnitude or more from the tissue surface to the skeleton. Light attenuation of photosynthetically active radiation (PAR) within the tissue is wavelength dependent due to the presence of host pigments and symbiont photopigments, where the blue spectral region is attenuated to a larger extent than red light (Wangpraseurt et al. 2012). Substantial distortions of photosynthesis vs. irradiance curves and photosynthetic action spectra can thus be observed when relating coral photosynthesis to incident irradiance instead of the actual scalar irradiance (Kühl et al. 1995).

In this study, we determine the first spectrally-resolved measurements of $\sigma_{\text {II }}(\lambda)$ in an important reef building coral species, Pocillopora damicornis. We subsequently evaluate these properties alongside measurements of the light microenvironment within the coral tissues to assess the role of tissue optics in affecting light availability and hence $\sigma_{\|}(\lambda)$. These data are finally applied to yield absolute electron transport rates based on calculations of the effective light field in coral tissue.

\section{Methods}

166

\subsection{Experimental material}



depth) of Heron Island, in the southern Great Barrier Reef of Australia $\left(151^{\circ} 55^{\prime} \mathrm{E}, 23^{\circ} 27^{\prime} \mathrm{S}\right)$ and were transported to the coral holding facility of the University of Technology Sydney. Here, all colonies were maintained in tanks provided with artificial seawater (prepared with 'Ocean Nature' sea salt,

172 Aquasonic) that was circulated through a natural biological sump. Tanks were provided with an incident photon irradiance of 50-70 $\mu \mathrm{mol}$ photons $\mathrm{m}^{-2} \mathrm{~s}^{-1}$ (250 $\mathrm{W}$ metal-halide lamps, Aqualine, Aqua Medic Inc., colour temperature $13,000 \mathrm{~K}$ ) set to a $14 \mathrm{~h}: 10 \mathrm{~h}$ day:night cycle at a constant temperature of $26^{\circ} \mathrm{C}$. Corals were fragmented into nubbins with a surface area of $\sim 1 \mathrm{~cm}^{2}$ two days prior to the experiment and kept in the same aquaria under essentially the same light conditions. isolated from Heron Island, in the southern Great Barrier Reef of Australia) was cultured in $f / 2$ medium (Guillard et al. 1975) in Erlenmeyer flasks under a downwelling photon irradiance of 30-40 $\mu \mathrm{mol}$ photons $\mathrm{m}^{-2} \mathrm{~s}^{-1}$ (TLD 18W/54 fluorescent tubes, Philips, colour temperature 10,000K) using the same irradiance cycle and water temperature as per the coral specimens. Cultures were maintained in exponential growth phase for 14-18 days prior to harvesting. Comparative measurements of $\sigma_{\| I}(\lambda)$ were performed on freshly isolated Symbiodinium cells from the tissues of $P$. damicornis (Hill et al. 2009). However, as the freshly isolated symbionts exhibited suboptimal $\Delta F / F_{M}$ values that were $\sim 20 \%$ lower than in cultured or in hospite Symbiodinium (data not shown), we propose that these preparations cannot be precisely used as a reference $\sigma_{\|}(\lambda)$ for Symbiodinium $\mathrm{sp}$. in our calculations.

\subsection{Experimental setup}

Variable chlorophyll fluorescence measurements were performed with a MC-PAM (Heinz-Walz

$\mathrm{GmbH}$, Effeltrich, Germany) equipped with either (i) an optical unit designed for leaf measurements (MCP-BK, Heinz-Walz GmbH) for measurements on intact coral nubbins, or (ii) an optical unit with a central quartz cuvette (ED-101US/MD) for measurements on cell suspensions of Symbiodinium sp. In the case of the MCP-BK optical unit the optical guide rod (Perspex) for fluorescence excitation points to the specimen almost perpendicularly $\left(12^{\circ}\right.$ angle of incidence) and tapers from $10 \times 10 \mathrm{~mm}$ at the emitter site to $6 \times 6 \mathrm{~mm}$ at the measuring point. The optical guide rod (Perspex) for fluorescence 
incidence. The optical unit ED-101US/MD consists of a centrally located place for a $10 \times 10 \mathrm{~mm}$ cuvette and perpendicularly positioned ports for the optical guide rods for the emitter and detector heads of the MC-PAM. Coral nubbins were housed in the measuring chamber (a glass cuvette with dimensions of $45 \times 40 \times 10 \mathrm{~mm}$ ), which was placed adjacent to the LED light source in the leaf clip setup. The position of coral nubbins within the chamber was carefully adjusted with a micromanipulator (MM33, Märzhäuser $\mathrm{GmbH}$, Wetzlar, Germany) in order to place a nubbin directly in the light path of the fluorometer. Throughout the measurements, coral nubbins were kept in the measuring chamber filled with seawater under continuous aeration provided by a glass Pasteur pipette attached to an air pump (Precision 9500, Aqua One, Ingleburn NSW, Australia). The aeration was adjusted to $\sim 3$ bubbles/s with a bleed valve that was inserted in the aeration line from the air pump to the measuring cuvette. The temperature was kept constant at $26^{\circ} \mathrm{C}$ using a temperature control unit (US-T, Heinz-Walz GmbH, Effeltrich, Germany). Symbiodinium cultures were kept in the quartz cuvette of the ED-101US/MD unit under continuous stirring using a small magnetic stir bar (Heinz-Walz GmbH, Effeltrich, Germany).

\subsection{Establishment of PAR lists}

A full set of PAR lists contains data of photosynthetically active radiation (PAR; $\mu$ mol photons $\mathrm{m}^{-2}$ $\left.\mathrm{s}^{-1}\right)$, for all wavelengths and intensity settings of actinic illuminations $(A L)$ and multiple turnover flashes (MT). As the three-dimensional curved geometry of the coral nubbins cannot be compared to the flat surface of a leaf, the PAR lists normally established by using a downwelling quantum irradiance sensor for leaves (US-MQS, Walz GmbH) were not applicable in our study. Moreover, we found considerable light inhomogeneity along the measuring head (see below), which would likely result in an incorrect estimation of incident PAR. Therefore, a quantum scalar irradiance sensor (US-SQS, Walz GmbH) was used to cross-calibrate the US-MQS sensor used to establish PAR list for the optical unit MCP-BK as follows. Both sensors were placed in the same orientation as for the sample holder used to calibrate PAR list during leaf measurements. The US-MQS sensor was placed in the leaf clip and into the middle of the emitter-detector Perspex rod pair, which serves as a light guide from the "Chip-On-Board" excitation source in the emitter unit to the photodiode in the detector unit. The light-collecting tip of the US-SQS sensor was carefully placed in the same location using a micromanipulator (MM33, Märzhäuser $\mathrm{GmbH}$, Wetzlar, Germany). Using $440 \mathrm{~nm}$ actinic light (AL) at 
setting 20, and with both sensors kept at $1 \mathrm{~mm}$ distance from the centre of the emitter-detector light guide pair, a photon irradiance of $2730 \mu \mathrm{mol}$ photons $\mathrm{m}^{-2} \mathrm{~s}^{-1}$ was measured by both sensors. Placing the US-SQS sensor in the same measurement configuration in the optical glass cuvette filled with seawater, the sensor was further carefully adjusted with the micromanipulator to different positions relative to the emitter light guide rod: the centre, the four corners, the edge, and the middle of the emitter and detector light guide rods. The calibration factor of the US-SQS sensor was adjusted for the water immersion effect. The PAR list was then recorded at each position. Significant heterogeneities in the light field could be quantified across a 10x10 mm square, i.e., representing the area of coral nubbins that were later placed in front of the MC-PAM optical unit. The highest variance was observed when PAR lists at the edges of emitter and detector light guide rods were compared (differing by a factor of two). Therefore, to maximise the accuracy of incident PAR calibrations, a $3.5 \times 5 \mathrm{~mm}$ aperture was mounted in front of the optical unit and adjusted so that the PAR obtained via the calibration process remained homogeneous at $\sim 1580 \mu$ mol photons $\mathrm{m}^{-2} \mathrm{~s}^{-1}$ using $440 \mathrm{~nm} \mathrm{AL}$ at setting 20. The PAR list established in this way was used for all coral measurements in this study. PAR lists in the cuvette system were subsequently verified using a spherical micro-quantum sensor (US-SQS/WB, Heinz-Walz GmbH, Effeltrich, Germany) according to (Schreiber et al. 2012).

\subsection{Measurement protocol}

Coral nubbins and cell suspensions were dark-acclimated for $15 \mathrm{~min}$ in the presence of weak farred light (setting 2 on the MC-PAM) to ensure oxidation of the intersystem photosynthetic electron transport pool and obtain a defined reference state for chlorophyll fluorescence measurements of the PSII maximum photochemical efficiency $\left(\Delta \mathrm{F} / \mathrm{F}_{\mathrm{M}}\right)$ and the PSII absorption cross-section $\left(\sigma_{\mathrm{II}}(\lambda)\right) . \Delta \mathrm{F} / \mathrm{F}_{\mathrm{M}}$ was quantified via application of a strong saturating light pulse (intensity: 3500-4000 $\mu$ mol photons $\mathrm{m}^{-2}$ $\mathrm{s}^{-1}$, width: $0.6 \mathrm{~s}$ ), followed by a dark acclimation period of $1 \mathrm{~min}$. A pre-programmed script optimised for coral nubbins (see below 'Determination of functional absorption cross-section of PSIl') was then applied according to Schreiber and Klughammer (2013) to determine $\sigma_{\| I}(\lambda)$ sequentially for five wavelengths: $440,480,540,590$ and $625 \mathrm{~nm}$. A further 5 min of dark-acclimation in the presence of weak far-red was applied to ensure full oxidation of the PQ-pool followed by an automated steadystate light curve (SSLC) with 3 min incubation at each irradiance to examine the light response of the 
PSIl photochemical efficiency $\left(\Delta \mathrm{F}^{\prime} / \mathrm{F}_{\mathrm{M}}{ }^{\prime}\right)$ of the effective PSIl quantum yield. The SSLC comprised of a

257

258

259

260

261

262

263

264

265

266

267

268

269

270

271

272

273

274

275

276

277

278

279

280

281

282

283

284 series of increasing irradiance of a specific wavelength (here the $440 \mathrm{~nm}$ LED), with data recorded via Light Curve Program files (Icp-files, Schreiber et al. 2012). The lowest irradiance levels $(<10 \mu \mathrm{mol}$ photons $\mathrm{m}^{-2} \mathrm{~s}^{-1}$ ) were generated by using the MC-PAM measuring light (ML) at high pulse frequency settings (1000-2000), where the same colour ML was used as per the AL. Three replicates were run using a new nubbin for each measurement. Data are thus shown as averages \pm standard error (S.E.) of three independent biological replicates of $P$. damicornis nubbins.

\subsection{Measurements of wavelength-dependent absorption cross-section of PSII}

A pre-programmed fast kinetic trigger file of the MC-PAM system (Sigma500.FTM) was used for automated measurements of fast fluorescence induction kinetics according to Schreiber and Klughammer (2013). Consecutive $O-I_{1}$ rise kinetics were recorded for each LED colour (440, 480, 540, 590 and $625 \mathrm{~nm}$ ) using pre-programmed script files, which define wavelength-specific intensities of $\mathrm{ML}$ and $\mathrm{AL}$ (or multiple turnover light pulses), as well as the amplifier gain and the time intervals between the start of various trigger files (these scripts are available from the primary author upon request).

The optimisation process for obtaining reliable $\sigma_{\| I}(\lambda)$ values for nubbins of $P$. damicornis consisted of two steps. Firstly, the ML intensity and gain were adjusted to obtain similar minimal fluorescence $\left(F_{0}\right)$ values, which facilitate a comparison of the rise kinetics. Secondly, the intensity of actinic light or multiple turnover light pulses were adjusted to obtain similar initial rise kinetics of the $\mathrm{O}-\mathrm{I}_{1}$ curves for all colours. When initial slopes are identical, differences in PAR are directly proportional to changes in $\sigma_{\| I}(\lambda)$. The $\sigma_{\| I}(\lambda)$ values were derived using a dedicated fitting routine provided by the PamWin-3 software (Heinz-Walz GmbH, Effeltrich, Germany) according to Schreiber et al. (2012). The fitting is based on the reversible radical pair model of PSII originally described by Lavergne and TrissI (1995) extended to take into account $Q_{A}{ }^{-}$reoxidation (Schreiber et al. 2012). The free-fitting parameters in this model that are fitted by the PamWin-3 program, are i) $\mathrm{J}$, a connectivity parameter (Lavergne and Trissl 1995), ii) $\tau$, the time constant of light-driven reduction of $Q_{A}$ (by actinic light or multiple turnover pulses), and iii) $\tau$ (reox), the time constant of $Q_{A}{ }^{-}$reoxidation. Additional parameters $F_{0}$ and $I_{1}\left(O\right.$ and $I_{1}$ levels in the PAM fluorescence induction terminology) are directly measured to define the variable 
fluorescence $(\Delta \mathrm{F})$ that can be induced by a combination of strong continuous light $(\mathrm{AL})$ or multiple turnover pulse (MT) and a single turnover saturating flash (ST pulse). The measurement is routinely carried out in the presence of weak far-red light (peaking at $735 \mathrm{~nm}$ ), i.e., when the PQ pool is oxidised. The $\mathrm{O}-\mathrm{I}_{1}$ curves recorded with the five colours were fitted simultaneously with fixed $J$ and $\tau$ (reox) parameters. As $J$ and $\tau($ reox $)$ are intrinsic dynamic properties of PSII, they should not be influenced by the colour of light used for induction. Fluorescence was recorded at $\square 665 \mathrm{~nm}$ using a RG long-pass emission filter in front of the MCP-D detector head.

\subsection{Direct measures of light field within coral tissue}

To directly measure the scalar irradiance within coral tissue $\left(E_{0}\right.$ (meas)) we used scalar irradiance microsensors (Lassen et al. 1992) as described previously (Wangpraseurt et al. 2012). Briefly, coral fragments were placed in a black flow-through chamber supplied with seawater ( $25^{\circ} \mathrm{C}$; flow velocity $\sim 3 \mathrm{~cm} \mathrm{~s}^{-1}$ ) and illuminated with a downwelling photon irradiance of $400 \mu \mathrm{mol}$ photons $\mathrm{m}^{-2} \mathrm{~s}^{-1}$. Scalar irradiance was recorded within coenosarc tissue, i.e., the connective tissue in between polyps measuring light microprofiles from the skeleton surface upwards into the tissue surface. Prior to each scalar irradiance measurement, a micro-incision was made with a micro-needle (effective tip size $<50$ $\mu \mathrm{m}$; ProSciTech Pty Ltd, USA) to allow smooth sensor penetration through the tissue. Five replicate profiles were carried out on randomly chosen coenosarc tissues of $P$. damicornis. Spectral data was recorded with the scalar irradiance microsensors connected to a fibre-optic spectrometer (USB2000+, Ocean Optics, Dunedin, FL, USA) controlled by the manufacturer's software (Spectrasuite). Scalar irradiance measurements were normalised to the incident downwelling irradiance $\left(E_{d}\right)$, as measured with the microsensor positioned in the collimated light beam above a black non-reflective surface. al. (2012) as:

$$
\sigma_{I I}(\lambda)=\frac{1}{\tau \cdot N_{A} \cdot E_{d}}
$$


312 where $\sigma_{\mathrm{II}}(\lambda)\left(\mathrm{nm}^{2}\right)$ is the intrinsic wavelength-dependent functional absorption cross section of PSII (in

313 optically thin suspension) or the apparent wavelength-dependent functional absorption cross section

314 of PSII (in intact corals). Here, $\tau$ is the time constant of light-driven $\mathrm{Q}_{\mathrm{A}}{ }^{-}$reduction (ms) determined from

315 the fast fluorescence kinetics measurements (see above), $\mathrm{N}_{\mathrm{A}}=$ Avogadro's constant $\left(6.03 \cdot 10^{23}\right.$ quanta [mol photons] $\left.{ }^{-1}\right)$, and $E_{\mathrm{d}}\left(\mu \mathrm{mol}\right.$ photons $\left.\mathrm{m}^{-2} \mathrm{~s}^{-1}\right)$ is the incident downwelling irradiance that was kept equal in the coral and culture experiments.

We theoretically derived the effective scalar irradiance within the coral tissue by assuming that in first approximation any change in $\sigma_{\|}(\lambda)$ between coral and culture is caused by differences in the coral tissue scalar irradiance with respect to incident irradiance at a given wavelength, i.e., $\Delta \sigma_{\|}=\Delta \mathrm{E}$. Therefore we calculated the ratio of $\sigma_{\|}$(coral) $/ \sigma_{\|}$(suspension) at each wavelength $(440,480,540,590$ and $625 \mathrm{~nm})$ and subsequently used the average value $(\mathrm{n}=5-6)$ of the $\sigma_{\|}($coral $) / \sigma_{\|}($suspension) ratio to calculate the effective PAR at a given wavelength $(\lambda)$ as:

This equation is valid only if it is assumed that all cells involved in fluorescence measurements are exposed to the same scalar irradiance intensity. This assumption is likely only justified for the topmost layers of tissue which is monitored via short-wavelength fluorescence. However, if excited within deeper layers, the fluorescence signal is likely to become re-absorbed. We propose that potential errors in $\sigma_{\|}(\lambda)$ calculations are minimised when strongly-absorbed $440 \mathrm{~nm}$ light is used (Schreiber et al. 2011). Therefore, recordings of light response curves with determinations of photon absorption rate of PSII $\left(P A R_{\|}\right)$and electron turnover rate of PSII $\left(E T R_{\|}\right)$were carried out using $440 \mathrm{~nm}$ light (see below).

2.9. Photon absorption rate of PSII $\left(P A R_{\|}\right)$and electron turnover rate of $\mathrm{PSII}\left(E T R_{\|}\right)$

The wavelength-dependent quantum absorption rate of PSII (PAR , photons $\left.\mathrm{PSII}^{-1} \mathrm{~s}^{-1}\right)$, which is equivalent to the photosynthetically usable radiation, was calculated as 
344 where PAR is either $E_{\mathrm{d}}, E_{0}$ (calc) or $E_{0}$ (meas). The wavelength-dependent absolute electron turnover 345 rate of PSII, ETR ${ }_{\|}$was thus calculated as

$$
\operatorname{ETR}_{I I}=P A R_{I I} \cdot\left[\frac{\Delta F^{\prime} / F_{M}^{\prime}}{\Delta F / F_{M}}\right]
$$

where $\Delta F^{\prime} / F_{M}{ }^{\prime}$ is the effective quantum yield of PSIl (in the light-adapted state), and $\Delta F / F_{M}$ is the maximal quantum efficiency of PSII (determined in the dark-adapted state with weak far-red background light). The ETR parameters $E T R_{\|}{ }^{\max }$ and $E_{k}(I I)$ were determined using the curve fitting protocol of PamWin-3.

Optical absorption spectra for Symbiodinium $s p$. were recorded by using a custom built spectrophotometer composed of a Tungsten-Halogen light source (LS-1, Ocean Optics), and a fibre optic spectrophotometer (USB2000, Ocean Optics) interfaced with an integrating sphere (FOIS-1, Ocean Optics) (see details in Petrou et al. 2013). Symbiodinium cells were filtered to GF/F filter paper (Whatman) which was placed directly in the light path of the Tungsten-Halogen lamp and across the collection port of the integrating sphere Absorption spectra were recorded using Spectra Suite software (Ocean Optics). Reference spectra were taken on clean filter paper filtered only with f/2 medium. Spectra were averaged $(n=4)$ to increase $S / N$ ratio and the average spectrum is shown along with the standard error (S.E.) at 440, 480, 540, 590 and $625 \mathrm{~nm}$ (Fig. 1). for intact corals and cultures 

and in culture, with highest values in the blue and a progressive decrease towards the red end of the spectrum (Fig.1). However, the absolute values of $\sigma_{\|}$differed between intact corals and Symbiodinium culture with mean $\sigma_{\|}$values of $\sim 6.5$ and $\sim 4.5$ at $440 \mathrm{~nm}$ for cultured Symbiodinium cells and $P$. damicornis nubbins, respectively (Fig. 1). For $P$. damicornis, $\sigma_{\|}(\lambda)$ values were ca. $20-30 \%$ lower between $440-540 \mathrm{~nm}$ (paired t-test, $\mathrm{p}<0.05$ ), but ca. 15\% higher at $590 \mathrm{~nm}$ than for cultured Symbiodinium. However, it should be noted that these differences at $590 \mathrm{~nm}$ were not statistically significant $(\mathrm{p}<0.05)$. In contrast, mean $\sigma_{\|}$values in coral and culture were identical at $625 \mathrm{~nm}$ ( $p>0.05)$. The absorption spectrum of cultured Symbiodinium followed closely the shape of the $\sigma_{\| I}(\lambda)$ (Fig. 1), although the relative values differed when both spectra were normalised to their maximal levels. The corresponding data of the $\mathrm{O}-\mathrm{I}_{1}$ kinetic rise that were used for $\sigma_{\|}$calculations are shown in Table 1, which shows that largely different PAR values were adjusted at different wavelengths to attain nearly identical $\tau$ values.

\subsection{Effect of light microenvironment on $\sigma_{\|}(\lambda)$ of Symbiodinium}

Microscale spectral scalar irradiance at and within the tissue surface revealed strong spectral distortion from the incident downwelling irradiance $\left(E_{\mathrm{d}}\right)$, indicating that Symbiodinium in the intact coral and in suspension are subject to different light regimes (Fig. 2). Most pronounced was the redshifted enhancement in tissue scalar irradiance relative to $E_{\mathrm{d}}$. To assess whether differences in $\sigma_{\|}$ between coral and suspension translated to differences between the light field of the dilute suspension (i.e. $E_{\mathrm{d}}$ ) and the light field within the intact coral $\left[E_{0}(\right.$ meas) $]$ we also examined the ratio of $\sigma_{\|}($coral $) / \sigma_{\|}\left(\right.$suspension) vs. $E_{0}$ (meas) $/ E_{d}$ (Fig. 2). The relative differences in $\sigma_{\|}($coral $) / \sigma_{\|}($suspension) followed closely (5-10\% deviation) the differences in light availability for Symbiodinium in the intact coral vs. culture in the blue-green and red spectral region (Fig. 2). However, in the orange-red region (590-625 nm), the differences between coral and culture did not match well with measured differences in $\sigma_{\|}$(offset between PAR and $\sigma_{\|}$) of $\sim 20-40 \%$ (Fig. 2). 
Absolute electron transport rates under $440 \mathrm{~nm}$ light were determined for intact corals using full downwelling irradiance $\left(E_{\mathrm{d}}\right)$ or measured scalar irradiance within the tissue $\left(E_{0}(\mathrm{calc})\right)$ for PAR in equation 3 (Fig. 3). Based on $E_{d}$, the mean TTR $_{\|}{ }^{\max }$ was 370 electrons $\mathrm{PSII}^{-1} \mathrm{~s}^{-1}$ ( \pm 30 S.E.) and the mean $E_{k}(I I)$ was 402 ( \pm 37 S.E.) photons $\mathrm{PSII}^{-1} \mathrm{~s}^{-1}$. As expected, when based on $E_{0}$, both the mean $E T R_{\|}{ }^{\max }$ and $E_{k}(I I)$ were substantially lower at 261 electrons $\mathrm{PSII}^{-1} \mathrm{~s}^{-1}$ ( \pm 35 S.E.) and 248 ( \pm 33 S.E.) photons $\mathrm{PSII}^{-1} \mathrm{~s}^{-1}$, respectively. The 1.4 factor of difference between $E_{\mathrm{d}}$ and $E_{0}$ (calc) reflects the ratio of intrinsic $\sigma_{\|}$(measured with an optically thin suspension of Symbiodinium) and the apparent $\sigma_{\|}$ determined with the intact coral (see Table 1).

In most studies, rETR values are calculated with the unrealistic assumption that a constant fraction of incident PAR is absorbed by the sample, independent of the colour of applied light and the actual absorption of this light in the sample. Nevertheless, measurements of rETR have proven useful for many practical applications to analyse relative changes in photosynthetic performance in particular species of corals, as long as the wavelength of light available (and the fundamental absorption properties) remained constant. When rETR curves of the intact coral $P$. damicornis based on $E_{\mathrm{d}}$ were compared with rETR curves based on $E_{0}$ (calc) the LCs showed different maximal relative electron transport rates ( $\mathrm{rETR}$ max of $36 \pm 2$ and $26 \pm 2$, respectively) and an onset of saturation at different irradiance levels ( $E_{k}$ of $143 \pm 16$ and $91 \pm 11 \mu$ mol photons $\mathrm{m}^{-2} \mathrm{~s}^{-1}$, respectively; Fig. 4).

\section{Discussion}

Our data demonstrate the wavelength dependency of the absorption cross section of PSII for Symbiodinium in culture and in hospite. This comparison is essential to determine the effective

421 Symbiodinium light absorption properties in hospite that can be used to estimate absolute electron transport rates in intact corals. Our measurements of $\sigma_{\| I}(\lambda)$ confirm past accounts of the spectral dependency of absorption that were based solely on fluorescence excitation spectra of PSII absorption from various Symbiodinium genotypes (Hennige et al. 2009). 

spectral region relative to the blue spectral region, which is indicative of a strong absorption of peridinin, the main light harvesting carotenoid of Symbiodinium (Fig. 1). These findings are supported by the absorption spectrum of Symbiodinium cultures (Fig. 1) and are in good agreement with previous results showing the absorption and fluorescence excitation spectrum of various clades, i.e., the energy absorbed in the blue $(440-480 \mathrm{~nm})$ region is partitioned to PSII-specific absorption to a larger extent than in the orange-red region (590-625 nm) (Hennige et al. 2009).

This spectral dependency of $\sigma_{\|}(\lambda)$ is important when considering how Symbiodinium photosynthesis is driven by light quality. Several previous studies have shown that blue light affects coral photosynthetic parameters differently than red light (e.g. Kinzie et al. 1984; Levy et al. 2003; Mass et al. 2010). For example, Levy et al. (2003) used FRR fluorescence to show that blue light decreases the effective quantum yield to a greater extent than red light. These results correspond to the differences of $\sigma_{\| \mid}$in blue vs. red parts of the spectrum reported here (Fig. 1) and indicate that 440 $\mathrm{nm}$ light is $\sim 3.4$-fold more efficiently utilised than $625 \mathrm{~nm}$ light in Symbiodinium.

We found that $\sigma_{\|}(\lambda)$ of Symbiodinium harboured in $P$. damicornis showed a similar wavelengthdependency as the cultured Symbiodinium, but that the absolute $\sigma_{\|}(\lambda)$ values differed significantly between the two types of symbiont environment (Fig. 2). Such deviation of the apparent $\sigma_{\| I}(\lambda)$ of $P$. damicornis from the intrinsic $\sigma_{\| I}(\lambda)$ of a dilute Symbiodinium sp. is a result of the lowering of the mean effective PAR within the coral tissues which increases with extinction. Similarly, Schreiber and Klughammer (2013) observed lower $\sigma_{\| I}(\lambda)$ values for intact plant leaves (Dandelion) compared to green algal cultures (Chlorella) and suggested that reduced $\sigma_{I I}(\lambda)$ values are due to part of the incident light being absorbed within the leaf and that such attenuation is most pronounced at wavelengths of strong absorption, i.e., blue and red in higher plants chloroplasts. In a more recent study, a thorough characterisation of $\sigma_{\| I}(\lambda)$ in optically dilute and dense Chlorella suspensions showed that for optically dense cell suspensions, apparent $\sigma_{\| I}(\lambda)$ has to be distinguished from intrinsic $\sigma_{\| I}(\lambda)$, with the latter corresponding to the value best measured with an optically thin cell suspension (Klughammer and Schreiber, submitted to Photosynthesis Research). identical; however, $\sigma_{\|}(\lambda)$ values cannot be determined with the same precision as intrinsic $\sigma_{\|}(\lambda)$ values 
since the underlying $\mathrm{O}-\mathrm{I}_{1}$ kinetics are the sum of many different components, as induced by a gradient of light intensities experienced by all cells within the optically-dense sample that ultimately contribute to the fluorescence signal. As noted above, it may be assumed that the reduction of apparent $\sigma_{\| I}(\lambda)$ with respect to intrinsic $\sigma_{\| I}(\lambda)$ is a measure of the reduction of the mean effective PAR with respect to incident PAR for the cells where fluorescence is measured. Importantly the measured effective quantum yield is representative for the same population of cells. Thus the MC-PAM allows us to obtain estimates of paired values of wavelength-dependent effective PAR and $\Delta \mathrm{F}^{\prime} / \mathrm{F}_{\mathrm{M}}{ }^{\prime}$ under identical conditions, from which absolute electron transport rates can be calculated.

Our light microsensor measurements within the intact coral suggest that differences in apparent $\sigma_{\| I}(\lambda)$ between corals and Symbiodinium culture are mainly due to differences in the light microenvironment of in hospite zooxanthellae. In the blue part of the spectrum, relative differences in $\sigma_{\| I}(\lambda)$ between coral and culture correlated closely with respective differences in light availability (Fig. 3). However, towards the red part of the spectrum (590-625 nm), light availability itself was insufficient to explain the differences in $\sigma_{\| I}(\lambda)$ between coral and culture (Fig. 3). The reason for this mismatch remains unknown, but could be related to the configurational differences of microsensor and chlorophyll fluorescence measurements, as discussed in the following section.

Since fluorescence is measured by the MC-PAM predominantly from a defined subsurface layer of photosymbionts, which is thinner than the microsensor tip, there is an intrinsic difference between the theoretical scalar irradiance calculated from differences in $\sigma_{\| I}(\lambda)$ as compared to measured scalar irradiance values that will average over a larger tissue volume. In cases of very thin coral tissue, it cannot be ruled out that the small white light collecting sphere of the microsensor substitutes for a substantial part of tissue that normally contributes to light absorption, which would thus affect the tissue light field. However, in the case of very thin tissue, such potential measurement artefact may also be masked by increased diffuse reflectance of the tissue light field from the underlying coral skeleton. Other comparisons of calculated and measured scalar irradiance measurements indicate that such artefact is likely negligible in strongly scattering sediment (Kühl and Jørgensen 1994). Additional reasons explaining these discrepancies could be (i) the presence of host pigments that might have additional effects on coral photosynthesis e.g. due to spectral conversion (Dove et al. 2008, Smith et al. 2013), (ii) differences in the penetration depth of the PAM excitation light (i.e. measuring and actinic light), (iii) imbalance of excitation energy that arrives in PSII or PSI, 
which was shown to be wavelength dependent in Symbiodinium (Hennige et al. 2009) or iv) other factors that could lead to physiological differences of cultured and in hospite Symbiodinium such as differences in the physico-chemical microenvironment of free-living and symbiotic Symbiodinium cells.

Despite these differences in microsensor and $\sigma_{\| I}(\lambda)$ data, both approaches revealed the same magnitude of light attenuation in the blue and blue-green spectral region, which thus allowed for estimating absolute electron transport rates in the blue region. Absolute electron transfer rates at 440 $\mathrm{nm}$ based on $E_{0}$ (calc) and $E_{0}$ (meas) were similar and both were approximately $40 \%$ lower than the rates estimated when using $E_{d}$ (Fig. 3). Interestingly, an $E T R_{\|}{ }^{\text {max }}$ value of ca. 220-250 electrons $\mathrm{PSII}^{-1}$ $\mathrm{s}^{-1}$ corresponded to $P A R_{\|}$values (i.e. photon absorption rate) of ca. 1000 photons $\mathrm{PSII}^{-1} \mathrm{~s}^{-1}$, when $P A R_{\|}$was based on $E_{0}$ (calc) or $E_{0}$ (meas) (Fig. 3); $P A R_{\|}$of 1000 photons $\mathrm{PSII}^{-1} \mathrm{~s}^{-1}$ (= 1 photon $\mathrm{PSII}^{-1}$ $\mathrm{ms}^{-1}$ ) corresponds to a rate of $Q_{A}{ }^{-}$reduction of $\sim 1 \mathrm{~ms}^{-1}$ under reference conditions. Consequently, at the maximum steady state $E T R_{\|}$, PSII turns over approximately once for every 4 photons absorbed by PSII (equivalent to 1 electron transported by PSII per $4 \mathrm{~ms}$ ) reflecting the limiting step between PSII and $\mathrm{PSI}$ at the protolytic reaction between $\mathrm{PQH}_{2}$ and the $\mathrm{Cyt}_{6} / \mathrm{f}$ complex. Similar $E T R_{\|}{ }^{\text {max }}$ rates have been observed from the single-celled photosynthetic model organism Chlorella grown under a similar light environment (Schreiber et al. 2012). The similarity of $E T R_{\|}{ }^{\max }$ values in the intact coral and Chlorella in dilute suspension suggests that photosynthetic electron transport is limited by the same partial reaction, i.e., most likely the protolytic step between $\mathrm{PQH}_{2}$ and $\mathrm{Cyt} \mathrm{b}_{6} / \mathrm{f}$ (see above).

Together, these results have important implications for our understanding of coral photobiology. Firstly, the optical properties of the coral host environment strongly affect the photon absorption rate of Symbiodinium and secondly, the MC-PAM approach facilitates a reliable estimation of such rates in a non-invasive and rapid manner. Our results also have implications for the determination of the light saturation irradiance, $E_{k}$, commonly derived from rETR vs. irradiance measurements. For instance, we found that if coral optical properties are not taken into account, $E_{k}$ was overestimated by ca. $40 \%$ (Fig. 4), thus further highlighting the value of coral tissue optics data for improving accuracy of not only photosynthesis rates but also photosynthesis-irradiance parameterisation, i.e., estimates in line with earlier findings comparing $P$ vs. E curves and action spectra using downwelling versus scalar irradiance as measures of the actinic light levels in coral tissue (Kühl et al. 1995).

In conclusion, we resolved the wavelength dependency of the functional absorption crosssection of PSII in Symbiodinium and provided the first PAM-based measures of absolute electron 
515 transfer rates in intact corals. The optical properties of coral tissues have a central role in affecting the 516 photosynthetic performance of in hospite Symbiodinium and our study implies that knowledge of coral

517 tissue optics is needed to determine absolute electron transport rates in corals. A potential future 518 approach could see a combination of microfiber-PAM measurements over different wavelengths, 519 and/or multilayered fluorescence models (see e.g. Evans, 2009) to account for different light 520 penetration and thus different operational tissue volumes contributing to PAM measurements at 521 different wavelengths. Expanding the applicability of PAM-based fluorescence parameters to 522 investigate absolute electron transport is an important step towards an improved means to better 523 characterise and hence understand how coral productivity varies over space and time.

524

\section{Contributions}

526 MS, DW, AL, US, MK and PR designed the experiments, MS, DW and BT performed the experiments, 527 MS, DW, AL, US, DS and MK analysed and interpreted the data, MS and DW wrote the paper with 528 the contribution of all co-authors, PR, MK contributed to reagents/materials, analysis tools.

530 Acknowledgements

531 This work was supported by the Danish Council for Independent Research / Natural Science (MK) 532 and the Carlsberg Foundation (MK), the Australian Research Council (MS, PR) and a postgraduate 533 scholarship from the University of Technology, Sydney (DW). Lars F. Rickelt is thanked for construction of scalar irradiance microsensors.

References

537 Dove, S.G., Lovell, C., Fine, M., Deckenback, J., Hoegh-Guldberg, O.V.E., Iglesias-Prieto, R., 538 Anthony, K., 2008. Host pigments: potential facilitators of photosynthesis in coral symbioses. Plant Cell Environ. 31, 1523-1533.

540 Enriquez, S., Mendez, E.R., Iglesias-Prieto, R., 2005. Multiple scattering on coral skeletons enhances light absorption by symbiotic algae. Limnol. Oceanogr. 50, 1025-1032.

Evans, J. R., 2009. Potential errors in electron transport rates calculated from chlorophyll fluorescence as revealed by a multilayer leaf model. Plant Cell Physiol. 50, 698-706. 
Gorbunov, M.Y., Kolber, Z.S., Lesser, M.P., Falkowski, P.G., 2001. Photosynthesis and photoprotection in symbiotic corals. Limnol. Oceanogr. 46, 75-85.

Guillard, R.R.L., 1975. Culture of phytoplankton for feeding marine invertebrates, in: Smith, W.L. and Chanley, M.H. (Eds.), Culture of Marine Invertebrate Animals. Plenum Press, New York, pp. 26-60.

Hennige, S.J., Suggett, D.J., Warner, M.E., McDougall, K.E., Smith, D.J., 2009. Photobiology of Symbiodinium revisited: bio-physical and bio-optical signatures. Coral Reefs 28, 179-195.

Hennige, S.J., McGinley, M.P., Grottoli, A.G., Warner, M.E., 2011. Photoinhibition of Symbiodinium spp. within the reef corals Montastraea faveolata and Porites astreoides: implications for coral bleaching. Mar. Biol. 158, 2515-2526.

Hill, R., Larkum, A.W.D., Frankart, C., Kühl, M., Ralph, P.J., 2004. Loss of functional Photosystem II reaction centres in zooxanthellae of corals exposed to bleaching conditions: using fluorescence rise kinetics. Photosynth. Res. 82, 59-72.

Hill, R., Ulstrup, K.E., Ralph, P.J., 2009. Temperature induced changes in thylakoid membrane thermostability of cultured, freshly isolated, and expelled zooxhanthellae from scleractinian corals. B. Mar. Sci. 85, 223-244.

Hoegh-Guldberg, O., Mumby, P.J., Hooten, A.J., Steneck, R.S., Greenfield, P., Gomez, E., Harvell, C.D., Sale, P.F., Edwards, A.J., Caldeira, K., Knowlton, N., Eakin, C.M., Iglesias-Prieto, R., Muthiga, N., Bradbury, R.H., Dubi, A., Hatziolos, M.E., 2007. Coral reefs under rapid climate change and ocean acidification. Science 318, 1737-1742.

Iglesias-Prieto, R., Govind, N., Trench, R., 1993. Isolation and characterization of three membranebound chlorophyll-protein complexes from four dinoflagellate species. Phil. Trans. Royal Soc. Lon. Ser. B: Biol. Sci. 340, 381-392.

Kinzie, R.A., Jokiel, P.L., York, R., 1984. Effects of light of altered spectral composition on coral zooxanthellae associations and on zooxanthellae invitro. Mar. Biol. 78, 239-248.

Koblizek, M., Kaftan, D., Nedbal, L., 2001. On the relationship between the non-photochemical quenching of the chlorophyll fluorescence and the Photosystem II light harvesting efficiency. A repetitive flash fluorescence induction study. Photosynth. Res. 68, 141-152. 
Kolber, Z.S., Prasil, O., Falkowski, P.G., 1998. Measurements of variable chlorophyll fluorescence using fast repetition rate techniques: defining methodology and experimental protocols. Biochim. Biophys. Acta-Bioenerg. 1367, 88-106.

Kühl, M., Jørgensen, B.B., 1994. The light field of micro-benthic communities: radiance distribution and microscale optics of sandy coastal sediments. Limnol. Oceanogr. 39, 1368-1398.

Kühl, M., Cohen, Y., Dalsgaard, T., Jørgensen, B.B., Revsbech, N.P., 1995. Microenvironment and photosynthesis of zooxanthellae in scleractinian corals studied with microsensors for $\mathrm{O}_{2}, \mathrm{pH}$ and light. Mar. Ecol. Prog. Ser. 117, 159-172.

Lassen, C., Ploug, H., Jørgensen, B.B., 1992. A fiberoptic scalar irradiance microsensor - application for spectral light measurement in sediments. FEMS Microbiol. Ecol. 86, 247-254.

Lavergne, J., Trissl, H.W., 1995. Theory of fluorescence induction in Photosystem-II - derivation of analytical expressions in a model including exciton-radical-pair equlibrium and restricted energy-transfer bewteen photosynthetic units. Biophys. J. 68, 2474-2492.

Lesser, M.P., 2000. Depth-dependent photoacclimatization to solar ultraviolet radiation in the Caribbean coral Montastraea faveolata. Mar. Ecol. Prog. Ser. 192, 137-151.

Levy, O., Dubinsky, Z., Achituv, Y., 2003. Photobehavior of stony corals: responses to light spectra and intensity. J. Exp. Biol. 206, 4041-4049.

Ley, A.C., Mauzerall, D.C., 1982. Absolute absorption cross-sections for photosystem-II and the minimum quantum requirement for photosynthesis in Chlorella vulgaris. Biochim. Biophys. Acta 680, 95-106.

Marcelino, L.A., Westneat, M.W., Stoyneva, V., Henss, J., Rogers, J.D., Radosevich, A., Turzhitsky, V., Siple, M., Fang, A., Swain, T.D., Fung, J., Backman, V., 2013. Modulation of lightenhancement to symbiotic algae by light-scattering in corals and evolutionary trends in bleaching. PLOS One 8, e61492.

Mass, T., Kline, D.I., Roopin, M., Veal, C.J., Cohen, S., Iluz, D., Levy, O., 2010. The spectral quality of light is a key driver of photosynthesis and photoadaptation in Stylophora pistillata colonies from different depths in the Red Sea. J. Exp. Biol. 213, 4084-4091.

Oxborough, K., Moore, C.M., Suggett, D.J., Lawson, T., Chan, H.G., Geider, R.J., 2012. Direct estimation of functional PSII reaction center concentration and PSII electron flux on a volume 
basis: a new approach to the analysis of Fast Repetition Rate fluorometry (FRRf) data. Limnol. Oceanogr. Meth. 10, 142-154.

Petrou, K., Jimenez-Denness, I., Chartrand, K., McCormack, C., Rasheed, M., Ralph, P.J., 2013. Seasonal heterogeneity in the photophysiological response to air exposure in two tropical intertidal seagrass species. Mar. Ecol. Prog. Ser. 482, 93-106.

Ragni, M., Airs, R.L., Hennige, S.J., Suggett, D.J., Warner, M.E., Geider, R.J., 2010. PSII photoinhibition and photorepair in Symbiodinium (Pyrrhophyta) differs between thermally tolerant and sensitive phylotypes. Mar. Ecol. Prog. Ser. 406, 57-70.

Ralph, P.J., Gademann, R., Larkum, A.W.D., Schreiber, U., 1999. In situ underwater measurements of photosynthetic activity of coral zooxanthellae and other reef-dwelling dinoflagellate endosymbionts. Mar. Ecol. Prog. Ser. 180, 139-147.

Schreiber, U., 2004. Pulse-Amplitude (PAM) fluorometry and saturation pulse method, in: Papageorgiou, G., Govindjee (Eds.), Chlorophyll fluorescence: A signature of Photosynthesis. Kluwer Academic Publishers, Dordrecht, The Netherlands, pp. 279-319.

Schreiber, U., Klughammer, C., 2013. Wavelength-dependent photodamage to Chlorella investigated with a new type of multi-color PAM chlorophyll fluorometer. Photosynth. Res. 114, 165-177.

Schreiber, U., Klughammer, C., Kolbowski, J., 2011. High-end chlorophyll fluorescence analysis with the MULTI-COLOR-PAM. I. Various light qualities and their applications. PAM Application Notes 1, 1-19.

Schreiber, U., Klughammer, C., Kolbowski, J., 2012. Assessment of wavelength-dependent parameters of photosynthetic electron transport with a new type of multi-color PAM chlorophyll fluorometer. Photosynth. Res. 113, 127-144.

Smith, E.G., D'Angelo, C., Salih, A., Wiedenmann, J., 2013. Screening by coral green fluorescent protein (GFP)-like chromoproteins supports a role in photoprotection of zooxanthellae. Coral Reefs 32, 463-474.

Strasser, R., Govindjee, 1992. On the O-J-I-P fluorescence transient in leaves and D1 mutants of Chlamydomonas reinhardtii. Photosynth. Res. 34, 135-135. 
Suggett, D.J., MacIntyre, H.L., Geider, R.J., 2004. Evaluation of biophysical and optical determinations of light absorption by photosystem II in phytoplankton. Limnol. Oceanogr. Meth. 2, 316-332.

Suggett, D.J., Moore, C.M., Hickman, A.E., Geider, R.J., 2009. Interpretation of fast repetition rate (FRR) fluorescence: signatures of phytoplankton community structure versus physiological state. Mar. Ecol. Prog. Ser. 376, 1-19.

Suggett, D.J., Moore, C.M., Geider, R.J., 2011. Estimating aquatic productivity from active fluorescence measurements, in: Suggett, D.J., Prášil, O., Borowitzka, M.A. (Eds.), Chlorophyll a Fluorescence in Aquatic Sciences: Methods and Applications. Springer, The Netherlands, pp. 103-127.

Teran, E., Mendez, E.R., Enriquez, S., Iglesias-Prieto, R., 2010. Multiple light scattering and absorption in reef-building corals. Appl. Opt. 49, 5032-5042.

Wangpraseurt, D., Larkum, A.W.D., Ralph, P.J., Kühl, M., 2012. Light gradients and optical microniches in coral tissues. Front. Microbiol. 3, 316.

Wangpraseurt, D., Larkum, A.W.D., Franklin, J., Szabó, M., Ralph, P.J., Kühl, M., 2014. Lateral light transfer ensures efficient resource distribution in symbiont-bearing corals. J. Exp. Biol. 217, 489-498.

Warner, M.E., Lesser, M.P., Ralph, P.J., 2011. Chlorophyll fluorescence in reef building corals, in: Suggett, D.J., Prášil, O., Borowitzka, M.A. (Eds.), Chlorophyll a Fluorescence in Aquatic Sciences: Methods and Applications. Springer, The Netherlands, pp. 209-222. 
650 Table 1. Parameters to calculate $\sigma_{\mathrm{II}}(\lambda)$ values of $P$. damicornis and Symbiodinium sp. ${ }^{\mathrm{a}}$

\begin{tabular}{lllllllll}
\hline Wavelength & $\boldsymbol{F}_{0}$ & $\mathrm{I}_{1}$ & PAR & $\boldsymbol{P}$ & $\boldsymbol{J}$ & $\boldsymbol{\tau}$ & $\boldsymbol{\tau}($ reox $)$ & $\sigma_{\boldsymbol{I I}}$ \\
\hline$(\mathrm{nm})$ & $(\mathrm{V})$ & $(\mathrm{V})$ & $\left(\mu \mathrm{mol}\right.$ photons $\left.\mathrm{m}^{-2} \mathrm{~s}^{-1}\right)$ & & & $(\mathrm{ms})$ & $(\mathrm{ms})$ & $\left(\mathrm{nm}^{2}\right)$ \\
\hline
\end{tabular}

$651 \quad$ P. damicornis

$\begin{array}{lllllllll}440 & 1.192 & 2.267 & 1050 & 0.432 & 0.760 & 0.327 & 0.354 & 4.46 \\ 480 & 1.234 & 2.346 & 1227 & 0.432 & 0.760 & 0.338 & 0.354 & 3.60 \\ 540 & 1.436 & 2.678 & 2254 & 0.432 & 0.760 & 0.31 & 0.354 & 2.14 \\ 590 & 1.373 & 2.606 & 3074 & 0.432 & 0.760 & 0.325 & 0.354 & 1.47 \\ 625 & 1.547 & 3.043 & 3256 & 0.432 & 0.760 & 0.323 & 0.354 & 1.32\end{array}$

652 Symbiodinium sp.

$\begin{array}{rrrrrrrrr}440 & 1.104 & 2.584 & 942 & 0.529 & 1.124 & 0.283 & 0.499 & 6.23 \\ 480 & 1.022 & 2.098 & 1110 & 0.529 & 1.124 & 0.312 & 0.499 & 4.80 \\ 540 & 1.186 & 2.269 & 2112 & 0.529 & 1.124 & 0.315 & 0.499 & 2.50 \\ 590 & 1.082 & 2.159 & 4827 & 0.529 & 1.124 & 0.289 & 0.499 & 1.19 \\ 625 & 1.078 & 2.224 & 4373 & 0.529 & 1.124 & 0.285 & 0.499 & 1.33\end{array}$

653

$654{ }^{a}$ Representative data from consecutive measurements of the $\mathrm{O}-\mathrm{I}_{1}$ rise kinetics in Pocillopora 655 damicornis and Symbiodinium $s p . F_{0}$, minimal fluorescence obtained by measuring light when $Q_{A}$ was 656 completely oxidised; $\mathrm{l}_{1}$, fluorescence obtained upon actinic light+single turnover flash, when $Q_{A}$ was 657 completely reduced; PAR, applied PAR to attain constant $\tau$ values; $\tau$, time constant of $\mathrm{Q}_{\mathrm{A}}{ }^{-}$reduction; $658 \tau($ reox $)$, time constant of $Q_{A}$ reoxidation. $\tau$ curves were fitted separately for the five wavelengths, 659 whereas parameters of $\tau$ (reox) (time constant of $\mathrm{Q}_{\mathrm{A}}$ oxidation) and $J$ (connectivity) were fixed since 660 they are independent of the wavelength (see Materials and Methods). 


\section{Author contributions}

MS, DW, AL, US, MK and PR designed the experiments, MS, DW and BT performed the experiments, MS, DW, AL, US, DS and MK analysed and interpreted the data, MS and DW wrote the paper with the contribution of all co-authors, PR, MK contributed to reagents/materials, analysis tools. 
Click here to download high resolution image

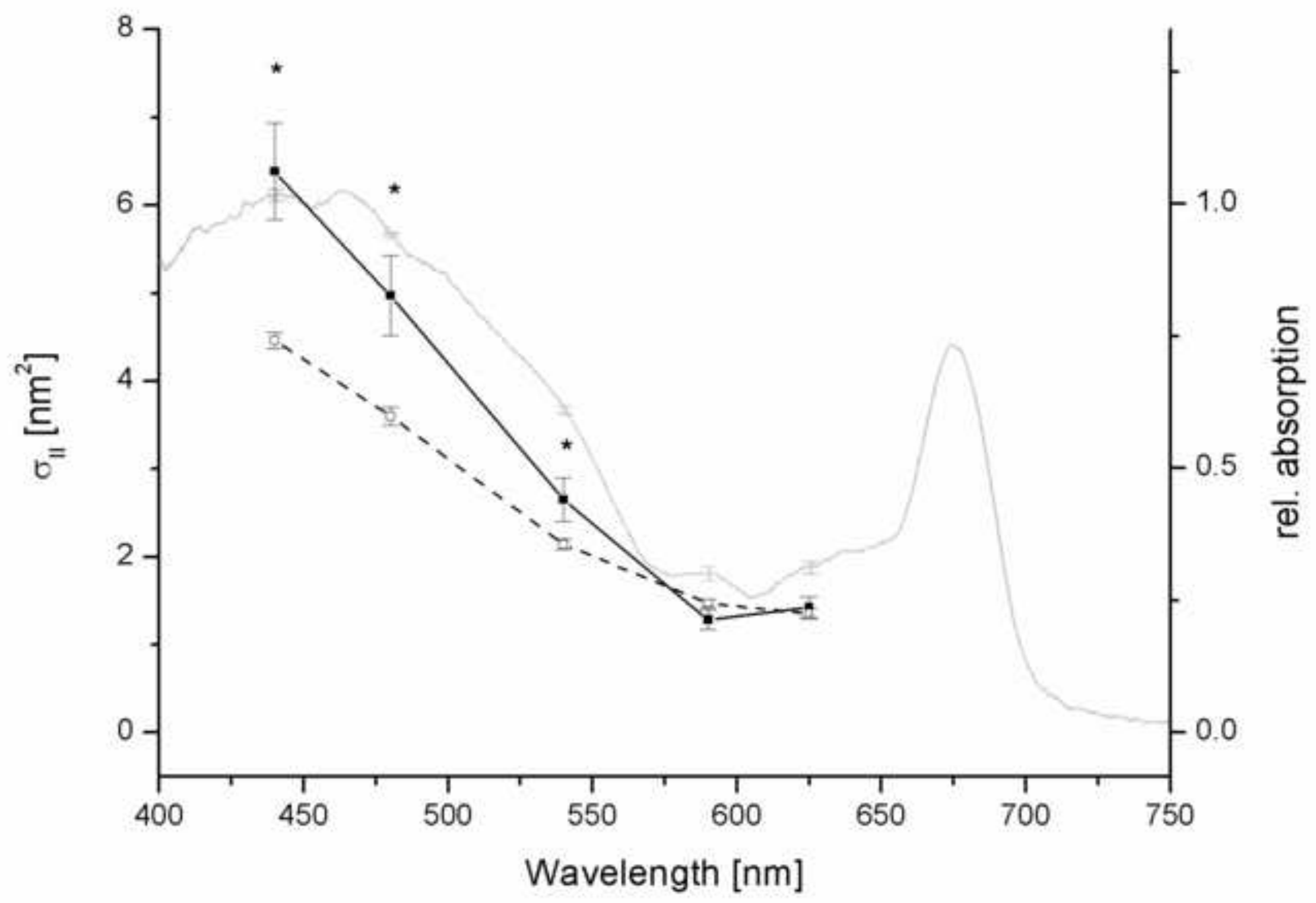


Click here to download high resolution image

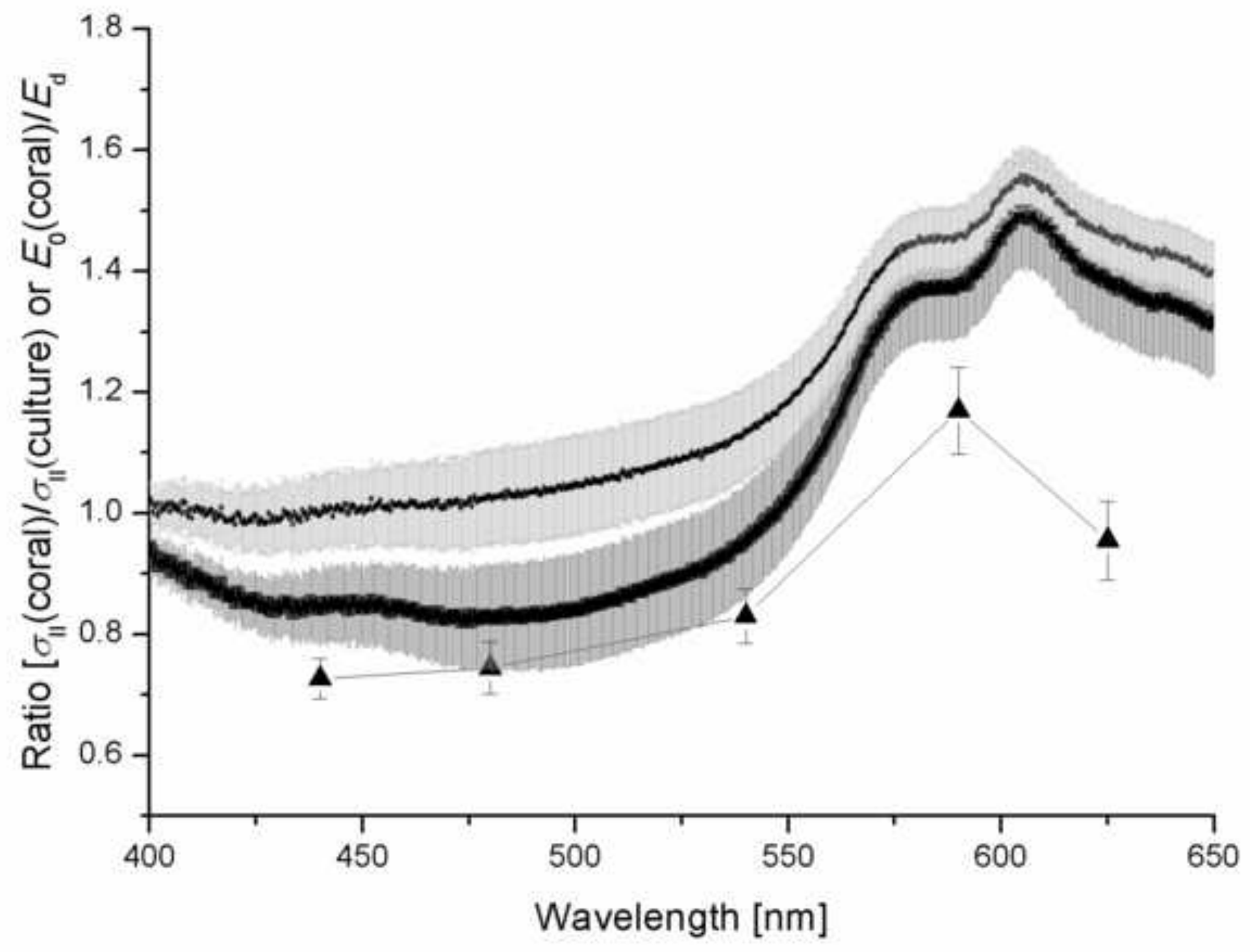


Click here to download high resolution image

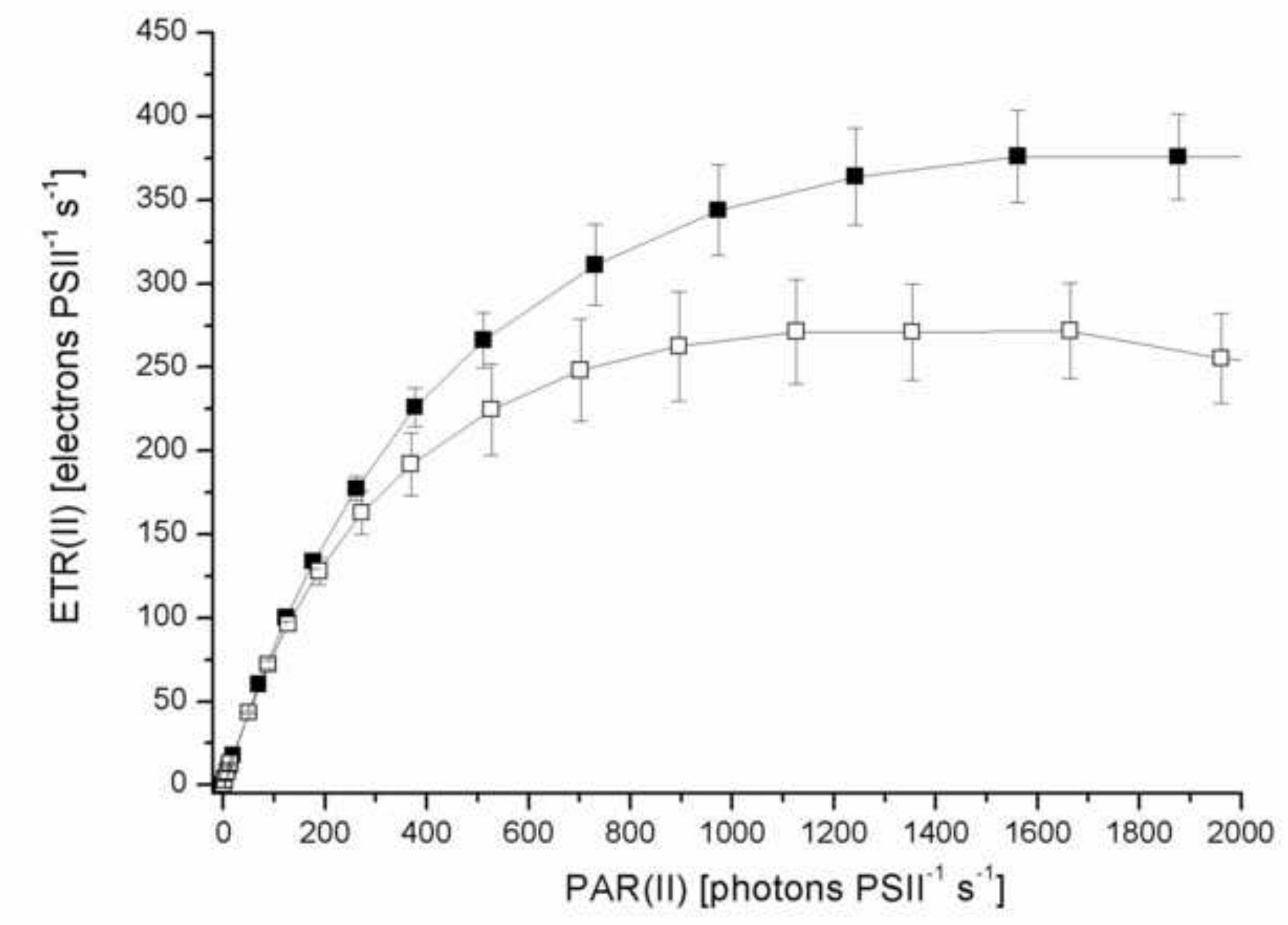

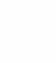


Click here to download high resolution image

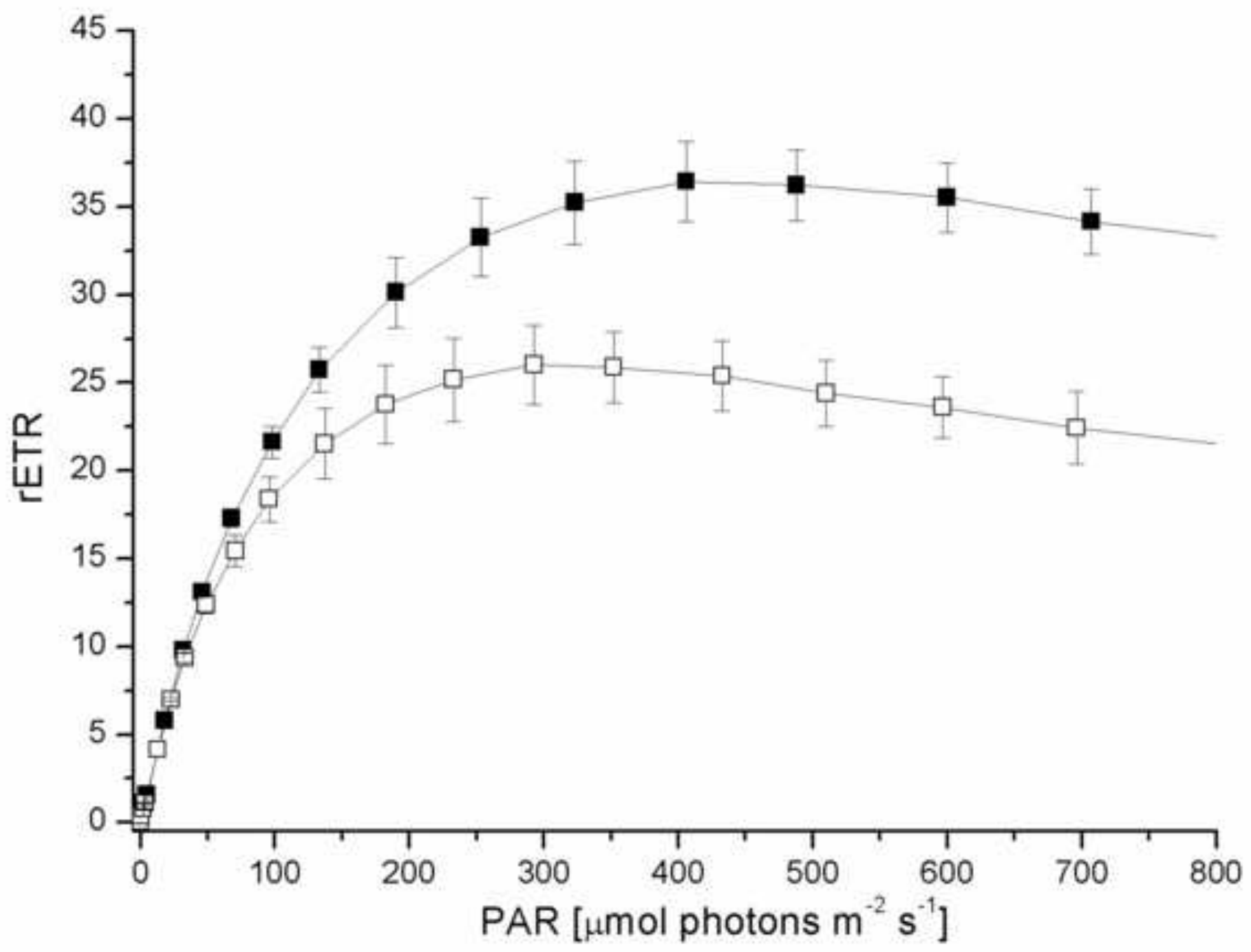




\section{Figure legends}

Fig. 1 Wavelength-dependent absorption cross section of PSII, $\sigma_{\|}(\lambda)$ in cultured Symbiodinium sp. cells (closed squares, solid line) and in coral Pocillopora damicornis (open squares, dashed line). $\sigma_{\text {III }}(\lambda)$ was derived from automated measurements of five consecutive $O-I_{1}$ rise curves for each colour in the presence of far-red background light. Each transient was averaged 5-10 times in order to improve the signal-to-noise ratio. The interval between consecutive $\mathrm{O}-\mathrm{I}_{1}$ measurements was $10 \mathrm{~s}$. $\sigma_{I I}(\lambda)$ values were derived by the PamWin-3 O-I fitting routine (Walz $\mathrm{GmbH}$, Effeltrich, Germany). Symbols and error bars represent the average \pm S.E. $(n=15) .{ }^{*}$ denotes statistically significant differences of $\sigma_{\text {III }}(\lambda)$ based on paired sample t-test $\left(\mathrm{P}_{\chi} 0.05\right)$. Relative absorption spectrum of Symbiodinium sp. (normalised to 1 at its maximum at $\sim 475 \mathrm{~nm}$, grey line). The spectrum is an average of 4 replicate measurements and S.E. is shown at $440,480,540,590$ and $625 \mathrm{~nm}$.

Fig. 2 Effect of coral optics on the ratios of $\sigma_{\text {III }}(\lambda)$ of Symbiodinium. The figure shows the ratio of $\sigma_{\|(\text {(coral) }} / \sigma_{\|(\text {(suspension) }}$ (triangles) vs. the ratio of scalar irradiance/incident downwelling irradiance at $80 \mu \mathrm{m}$ tissue depth (thick line) and at mean tissue depth (thin line). Standard errors (S.E.) of the scalar irradiance spectra at $80 \mu \mathrm{m}$ and at mean tissue depths and the $\sigma_{\|}(\lambda)$ ratios are shown in dark and light grey and black vertical lines, respectively

Fig. 3 Steady-state light curves of $E T R_{\|}$recorded at $440 \mathrm{~nm}$ of Pocillopora damicornis as a function of $P A R_{\|}$that was obtained based either on $E_{d}$ (closed squares) or on $E_{0}(c a l c)$ (open squares)

Fig. 4 Steady-state rel. ETR light curves recorded at $440 \mathrm{~nm}$ of Pocillopora damicornis as a function of either $E_{\mathrm{d}}$ (closed squares) or $E_{0}$ (calc) (open squares) 